\title{
On KMS Boundary Condition
}

\author{
By \\ Huzihiro Araki and Hideo Miyata*
}

\begin{abstract}
An invariant state satisfying the Kubo-Martin-Schwinger condition is studied. It is shown that the decomposition of a given state into extremal invariant states yields states satisfying the KMS boundary condition if and only if the cyclic representation associated with the given state is $\eta$-abelian, and that, if this is the case, the decomposition coincides with the standard central decomposition. The structure of the cyclic representation when it is non $\eta$-abelian is analyzed and typical examples are given. One of the examples gives a case where the cyclic representation is $G$-abelian but not $\eta$-abelian.
\end{abstract}

\section{$\S 1$. Introduction}

The Gibbs ensemble in quantum statistical mechanics satisfies the Kubo-Martin-Schwinger (KMS) boundary condition and a general property of such a state has been discussed [1], [2], [3], [4]. It is known that the center of the relevant $W^{*}$-algebra is time translation invariant [4]. From this it follows that the standard central decomposition yields again invariant states satisfying the KMS boundary condition. It is then an interesting question whether any further decomposition is possible and meaningful.

There exists theorems on the possibility of a unique decomposition into extremal invariant states under various assumptions: (weakly) asymptotic abelian [5], [6], $\eta$-abelian [7], $M$-abelian [8], large [9], G-abelian [10].

In this paper we shall show that, for an invariant state satisfying the KMS boundary condition, a decomposition into extremal invariant states is possible and yields exclusively states satisfying the KMS

Received July 9, 1968.

* Department of Physics, Kyoto University. 
boundary condition if and only if the cyclic representation associated with the given state is $\eta$-abelian. If that is the case, the decomposition coincides with the standard central decomposition.

If the restriction of the representation to the subspace of invariant vectors is abelian ( $G$-abelian), then any decomposition finer than the central decomposition is shown to yield a state not satisfying the KMS boundary condition. If the restriction of the representation to the subspace of invariant vectors is a factor, then it must be a finite factor. If it is type I, the structure of the state is in a sense completely analyzed. It is a tensor product of two spaces, where the time translation acts only on one space in which the invariant vector is unique and on the other space the state is a trace. If the given state satisfies the KMS boundary condition, then the part of the representation algebra in the first space does not contain any time translation invariant observable. The general case is a combination of abelian and factor situations. Typical examples for the two cases are given. In particular, we have an example where the system is (non trivially) $G$-abelian but not $\eta$-abelian and the state is a factor state.

From the analysis of the present paper, we have the impression that the central decomposition is probably optimal "good" decomposition for states satisfying the KMS boundary condition, as was hinted in [11], footnote to proposition 4. If the stationary observables are not commutative, a further non-unique reduction is possible according to their expectation values, but this does not change the structure of time dependent part.

\section{\$2. Preliminaries}

Let $\tau$ be a continuous representation of a locally compact group $G$ by automorphisms of a $C^{*}$-algebra $\mathscr{A}$ and $\phi$ be a $\tau(G)$ invariant state of $\mathfrak{A}$. Let the Hilbert space $H_{\varphi}$, the representation $\pi_{\varphi}$ of $\mathfrak{A}$, the cyclic unit vector $\Omega_{\varphi}$ and the continuous unitary representation $U_{\varphi}(G)$ of $G$ be canonically associated with $\phi$, namely 
(2.1) $\quad \varphi(A)=\left(\Omega_{\varphi}, \pi_{\varphi}(A) \Omega_{\varphi}\right), \quad A \in \mathfrak{U}$

(2.2) $\quad U_{\varphi}(g) \pi_{\varphi}(A) \Omega_{\varphi}=\pi_{\varphi}(\tau(g) A) \Omega_{\varphi}, \quad A \in \mathfrak{A}, g \in G$.

Let $E_{0}$ be the projection on the subspace of $H_{\varphi}$ consisting of all $U_{\varphi}(G)$ invariant vectors. Let

$$
\begin{aligned}
& R_{1}=\left(\pi_{\varphi}(\mathfrak{U}) \cup U_{\varphi}(G)\right)^{\prime \prime} \\
& R_{2}=\left(\pi_{\varphi}(\mathfrak{U})^{\prime} \cup U_{\varphi}(G)\right)^{\prime \prime} \\
& C_{1}=R_{1}^{\prime} \\
& C_{2}=R_{2}^{\prime} \\
& C_{0}=R_{1}^{\prime} \cap R_{2}^{\prime} .
\end{aligned}
$$

The set of all $\tau(G)$ invariant central elements of $\pi(\mathfrak{A})^{\prime \prime}$ is $C_{0}$.

Theorem 2. 1. The following equalities for von Neumann algebras on $E_{0} H_{\varphi}$ hold:

$$
\begin{aligned}
& E_{0}\left(E_{0} \pi_{\varphi}(\mathfrak{U}) E_{0}\right)^{\prime}=\left(E_{0} R_{1} E_{0}\right)^{\prime} E_{0}=R_{1}^{\prime} E_{0} \\
& \quad=E_{0} \pi_{\varphi}(\mathfrak{Q})^{\prime} E_{0}=E_{0} R_{2} E_{0} \\
& E_{0} \pi_{\varphi}(\mathfrak{U})^{\prime \prime} E_{0}=E_{0} R_{1} E_{0}=\left(E_{0} \pi_{\varphi}(\mathfrak{A})^{\prime} E_{0}\right)^{\prime} E_{0} \\
& \quad=\left(E_{0} R_{2} E_{0}\right)^{\prime} E_{0}=R_{2}^{\prime} E_{0} .
\end{aligned}
$$

Proof. Because of $U_{\varphi}(g) E_{0}=E_{0}$ and $U_{\varphi}(g) \pi_{\varphi}(\mathfrak{U}) U_{\varphi}(g)^{-1}=\pi_{\varphi}(\mathfrak{U})$ for $g \in G$, we have

$$
E_{0} R_{1} E_{0}=\left(E_{0} \pi_{\varphi}(\mathfrak{H})^{\prime \prime} E_{0}\right)^{\prime \prime} E_{0} .
$$

Since $U_{\varphi}(g) R U_{\varphi}(g)^{-1}=R$ for $R=\pi_{\varphi}(\mathfrak{A})$ implies the same for $R=\pi_{\varphi}(\mathfrak{A})^{\prime}$,

$$
E_{0} R_{2} E_{0}=\left(E_{0} \pi_{\varphi}(\mathfrak{U})^{\prime} E_{0}\right)^{\prime \prime} E_{0} .
$$

Since $E_{0}$ belongs to $R_{1}$ and $R_{2}$, these are von Neumann algebras and

$$
\begin{aligned}
& \left(E_{0} R_{1} E_{0}\right)^{\prime} E_{0}=R_{1}^{\prime} E_{\mathrm{v}} \\
& \left(E_{0} R_{2} E_{0}\right)^{\prime} E_{0}=R_{2}^{\prime} E_{0} .
\end{aligned}
$$

Since $R_{1}$ contains $\pi_{\varphi}(\mathfrak{U}), R_{1}^{\prime}$ is contained in $\pi_{\varphi}(\mathfrak{U})^{\prime}$ and

$$
E_{0} \pi_{\varphi}(\mathfrak{A})^{\prime} E_{0} \supset R_{1}^{\prime} E_{0} \text {. }
$$

Finally, let $A \in \pi_{\varphi}(\mathfrak{A})^{\prime \prime}, B \in \pi_{\varphi}(\mathfrak{A})^{\prime}, g \in G$. We have $U_{\varphi}(g) \pi_{\varphi}(\mathfrak{A}) U_{\varphi}(g)^{-1}$ $=\pi_{\varphi}(\mathfrak{l})$ and hence $U_{\varphi}(g) \pi_{\varphi}(\mathfrak{l})^{\prime} U_{\varphi}(g)^{-1}=\pi_{\varphi}(\mathfrak{l})^{\prime}$. Hence 


$$
\left[\pi_{\varphi}(A), \pi_{\varphi}(\tau(g) B)\right]=0 .
$$

Let $\psi_{1}, \psi_{2} \in E_{0} H_{\varphi}$. Then we have, from (2.15)

$$
\left(\psi_{1}, \pi_{\varphi}(A) U_{\varphi}(g) \pi_{\varphi}(B) \psi_{2}\right)=\left(\psi_{1}, \pi_{\varphi}(B) U_{\varphi}\left(g^{-1}\right) \pi_{\varphi}(A) \psi_{2}\right) 。
$$

Taking the Godement mean and using the mean ergodic theorem [8], we have

$$
\left(\psi_{1}, \pi_{\varphi}(A) E_{0} \pi_{\varphi}(B) \psi_{2}\right)=\left(\psi_{1}, \pi_{\varphi}(B) E_{0} \pi_{\varphi}(A) \psi_{2}\right) .
$$

Hence

$$
\left[E_{0} \pi_{\varphi}(A) E_{0}, E_{0} \pi_{\varphi}(B) E_{0}\right]=0
$$

Therefore

$$
E_{0} \pi_{\varphi}(\mathfrak{U})^{\prime} E_{0} \subset\left(E_{0} \pi_{\varphi}(\mathfrak{H}) E_{0}\right)^{\prime} .
$$

From (2.19), (2.14), (2.12) and the commutant of (2.10), we have (2.8). A similar calculation yields (2.9).

Theorem 2.2. A state $\varphi$ is extremal in the set of all invariant states of $\mathfrak{A}$ if and only if $R_{1}^{\prime}$ is trivial (i.e. the set consisting of multiples of the identity operator).

Proof. Let $R_{1}^{\prime}$ contain a nontrivial projection $E$. Then $E \Omega_{\varphi} \neq$ $c \Omega_{\varphi}$ for any constant $c$ because $\pi_{\varphi}(\mathfrak{A}) \Omega_{\varphi}$ is dense in $H_{\varphi}$ and $E$ commutes with $\pi_{\varphi}(\mathfrak{U})$. Now

$$
\begin{aligned}
& \varphi_{1}=\left(\Omega_{\varphi}, \pi_{\varphi}(A) E \Omega_{\varphi}\right) /\left\|E \Omega_{\varphi}\right\|^{2} \\
& \rho_{2}=\left(\Omega_{\varphi}, \pi_{\varphi}(A)(1-E) \Omega_{\varphi}\right) /\left\|(1-E) \Omega_{\varphi}\right\|^{2}
\end{aligned}
$$

are $\tau(G)$ invariant states of $\mathfrak{A}$ and

$$
\begin{aligned}
& \varphi=\varphi_{1} \lambda+\varphi_{2}(1-\lambda) \\
& 1>\lambda=\left\|E \Omega_{\varphi}\right\|^{2}>0 .
\end{aligned}
$$

If $\varphi$ is extremal, then $\varphi_{1}$ must be $\varphi$, namely

$$
\left(\Omega_{\varphi}, \pi_{\varphi}(A) E \Omega_{\varphi}\right)=\left(\Omega_{\varphi}, \pi_{\varphi}(A) \Omega_{\varsigma}\right) \lambda .
$$

Since $\pi_{\varphi}(A) * \Omega_{\varphi}$ is dense in $H_{\varphi}$, we have $E \Omega_{\varphi}=\lambda \Omega_{\varphi}$, which is a contradiction. Hence $\varphi$ is extremal only if $R_{1}^{\prime}$ is trivial.

Next assume 


$$
\varphi=\lambda \varphi_{1}+(1-\lambda) \varphi_{2}, \quad 0<\lambda<1
$$

Then

$$
\varphi_{1}(A) \leqq \lambda^{-1} \varphi(A)
$$

for all $A \geqq 0$. Hence by a standard argument

$$
\varphi_{1}(A)=\left(\Omega_{\varphi}, \pi_{\varphi}(A) B \Omega_{\varphi}\right)
$$

where $B \in \pi_{\varphi}(\mathfrak{U})^{\prime}, B \geqq 0,\|B\| \leqq \lambda^{-1}$. If $\varphi_{1}$ is $\tau(G)$ invariant in addition, we have

$$
U_{\varphi}(g) B \Omega_{\varphi}=B \Omega_{\varphi}
$$

for $g \ni G$. This implies

$$
U_{\varphi}(g) B U_{\varphi}(g)^{-1}-B
$$

is 0 on $\Omega_{\varphi}$. Since $\pi_{\varphi}(\mathfrak{A}) \Omega_{\varphi}$ is dense in $H_{\varphi}$ and $(2.28)$ is in $\pi_{\varphi}(\mathfrak{A})^{\prime}$, we have

$$
U_{\varphi}(g) B U_{\varphi}(g)^{-1}=B .
$$

Namely $B \in R_{1}^{\prime}$. If $R_{1}^{\prime}$ is trivial, then $B=c \mathbf{1}$ and hence $\varphi_{1}=\varphi$. Therefore, if $R_{1}^{\prime}$ is trivial, $\varphi$ is extremal.

\section{§. KMS Boundary Condition}

In the rest of this paper, the group $G$ is the additive group of reals.

Definition 3. 1. A $\tau(G)$ invariant state $\varphi$ satisfies the KMS boundary condition if for any $\tilde{f}(p)$ of the class $\mathscr{D}$,

$$
\begin{aligned}
& \varphi\left(A B\left(f_{0}\right)\right)=\varphi\left(B\left(f_{\beta}\right) A\right) \\
& f_{\alpha}(t) \equiv \int d p \widetilde{f}(p) \exp (-i p[t+i \alpha]) \\
& B(f) \equiv \int(\tau(t) B) f(t) d t .
\end{aligned}
$$

Corollary 3.2. If a $G$ invariant state $\varphi$ satisfies the KMS boundary condition, then the center of $\pi_{\varphi}(\mathfrak{U})^{\prime \prime}$ is elementwise $G$ invariant ;

$$
U_{\varphi}(g) A U_{\varphi}(g)^{-1}=A \quad \text { if } \quad A \in \pi_{\varphi}(\mathfrak{R})^{\prime \prime} \cap \pi_{\varphi}(\mathfrak{A})^{\prime}
$$


Proof. See [4].

Corollary 3. 3. If a $G$ invariant state $\varphi$ satisfies the KMS boundary condition, then the vector state $\hat{\varphi}$ of $\pi_{\varphi}(\mathfrak{H})^{\prime \prime}$ defined by $\Omega_{\varphi}$ satisfies the KMS boundary condition relative to the $\tau(G)$ defined by $U_{\varphi}(g), g \in G$.

\section{Proof. See [4].}

Theorem 3.4. If a $\tau(G)$ invariant state $\varphi$ satisfies the KMS boundary condition, then the following conclusions hold: (1) $\Omega_{\varphi}$ is a trace vector for $R_{1}^{\prime}, R_{2}^{\prime}, E_{0} \pi_{\varphi}(\mathfrak{U})^{\prime \prime} E_{0}$, and $E_{0} \pi_{\varphi}(\mathfrak{H})^{\prime} E_{0}$. (2) $\Omega_{\varphi}$ is a cyclic and separating vector for $E_{0} \pi_{\varphi}(\mathfrak{A})^{\prime} E_{0}$ and for $E_{0} \pi_{\varphi}(\mathfrak{A})^{\prime \prime} E_{0}$ on $E_{0} H_{\varphi}$ (3) $\Omega_{\varphi}$ is separating for $R_{1}^{\prime}$.

Proof. If $B \in R_{2}^{\prime} \subset \pi_{\varphi}(\mathfrak{A})^{\prime \prime}$, then $B(t)=B$. Further

$$
\int f_{\alpha}(t) d t=2 \pi \tilde{f}(0)
$$

is independent of $\alpha$. Hence by the KMS boundary condition for $\hat{p}$

$$
\hat{\rho}(A B)=\hat{\varphi}(B A), \quad A \in R_{2}^{\prime}, \quad B \in \pi(\mathfrak{U})^{\prime \prime}
$$

where $\hat{\mathcal{P}}$ is the normal extension of $\varphi$ to $\pi_{\varphi}(\mathfrak{U})^{\prime \prime}$.

In particular, $\hat{\rho}$ is a trace on $R_{2}^{\prime}$. Since $E_{0} \in\left(R_{2}^{\prime}\right)^{\prime}, E_{0} \Omega_{\varphi}=\Omega_{\varphi}$ and $E_{0} R_{2}^{\prime}=E_{0} \pi_{\varphi}(\mathfrak{U})^{\prime \prime} E_{0}, \hat{\rho}$ is a normal trace on $E_{0} \pi_{\varphi}(\mathfrak{U})^{\prime \prime} E_{0}$ with the trace vector $\Omega_{\varphi}$. Since $\Omega_{\varphi}$ is cyclic for $E_{0} \pi_{\varphi}(\mathfrak{H})^{\prime \prime} E_{0}$, it must be separating, and it must also be a cyclic and separating trace vector for the commutant of $E_{0} \pi_{\varphi}(\mathfrak{U})^{\prime \prime} E_{0}$ on $E_{0} H_{\varphi}$. Hence $\Omega_{\varphi}$ is a cyclic and separating trace vector for $E_{0} \pi_{\varphi}(\mathfrak{H})^{\prime} E_{0}=R_{1}^{\prime} E_{0}$ in $H_{\varphi}$. (See Theorem 2.1) Since $A \in R_{1}^{\prime} \rightarrow A E_{0} \in R_{1}^{\prime} E_{0}$ is an isomorphism, $\Omega_{\varphi}$ is also a faithful trace vector for $R_{1}^{\prime}$.

Theorem 3.5. For a $\tau(G)$ invariant state $\varphi$ satisfying the KMS boundary condition, the following conditions are equivalent:

(i) $R_{1}^{\prime}$ is trivial,

(ii) $\varphi$ is extremal among $\tau(G)$ invariant states of $\varphi$,

(iii) $\operatorname{dim} E_{0} H_{\varphi}=1$,

(iv) $\mathrm{M}\left(\pi_{\varphi}(\tau(t) A)\right)=\varphi(A) \mathbb{1}, A \in \mathfrak{A}$ where $\mathrm{M}$ is the Godement mean. 
The same equation holds if $\mathrm{M}$ is replaced by and invariant mean $\eta$.

Proof. The equivalence of (i) and (ii) is given in Theorem 2.2. If $R_{1}^{\prime}$ is trivial, then $E_{0} \pi_{\varphi}(\mathfrak{Q})^{\prime} E_{0}$ is trivial and has a cyclic (and separating trace) vector $\Omega_{\varphi}$ on $E_{0} H_{\varphi}$. Hence $E_{0} H_{\varphi}$ must be one dimensional. Hence (i) implies (iii). Conversely, if $E_{0} H_{\varphi}$ is one dimensional, $E_{0} R_{1}^{\prime}$ is trivial and hence $R_{1}^{\prime}$, which is isomorphic to $E_{0} R_{1}^{\prime}$, must be one dimensional. Hence (iii) implies (i). Finally we have

$$
\begin{aligned}
& \mathbb{M}\left\{\varphi\left(B_{1}[\tau(t) A] B_{2}\left(f_{0}\right)\right)\right\} \\
= & \mathbb{M}\left\{\varphi\left(B_{2}\left(f_{\beta}\right) B_{1} \tau(t) A\right\}\right. \\
= & \left(\Omega_{\varphi}, \pi_{\varphi}\left(B_{2}\left(f_{\beta}\right) B_{1}\right) E_{0} \pi_{\varphi}(A) \Omega_{\varphi}\right) \\
= & \varphi\left(B_{2}\left(f_{\beta}\right) B_{1}\right) \varphi(A)=\varphi\left(B_{1} B_{2}\left(f_{0}\right)\right) \varphi(A)
\end{aligned}
$$

where we have used (iii) in the last step. Hence (iii) implies (iv). Conversely, (iv) implies that $\pi_{\varphi}(\mathfrak{U})$ is $\eta$-abelian. It is known that if $\pi_{\varphi}(\mathfrak{l})$ is $\eta$-abelian, then the weak cluster property (iv) implies (i), (ii), (iii).

Theorem 3.6. Let $\mathfrak{A}$ be separable. In order that a $\tau(G)$ invariant state $\varphi$ satisfying KMS boundary condition is an integral of a family of $\tau(G)$ extremal invariant states satisfying KMS boundary condition, it is necessary and sufficient that $\pi_{\varphi}(\mathfrak{U})$ is $\eta$-abelian. If $\pi_{\varphi}(\mathfrak{H})$ is $\eta$-abelian, the central decomposition of the state $\varphi$ coincides with the decomposition of $\varphi$ into extremal $G$ invariant states.

Proof. If $\pi_{\varphi}(\mathfrak{U})$ is $\eta$-abelian, then any $\tau(G)$ invariant factor state of $\pi_{\varphi}(\mathfrak{U})$ is extremal. On the other hand, the KMS boundary condition implies that the center of $\pi_{\varphi}(\mathfrak{U})^{\prime \prime}$ commutes with $U_{\varphi}(t)$ and hence it is contained in $R_{1}^{\prime}$. Therefore, if $\pi_{\varphi}(\mathfrak{U})$ is $\eta$-abelian and $\varphi$ satisfies the KMS boundary condition, then $\varphi$ is a factor state if and only if it is an extremal invariant state.

Now consider a general state $\varphi$, which satisfies the KMS boundary condition. Let $F$ be any central projection and consider the new state

$$
\varphi_{F}(A) \equiv\left(\Omega_{\varphi}, \pi_{\varphi}(A) F \Omega_{\varphi}\right) /\left\|F \Omega_{\varphi}\right\|^{2}
$$


Then $\varphi_{F}$ is $\tau(G)$ invariant because $F$ commutes with $U_{\varphi}(t)$. Further, since $\pi_{\varphi}(A)^{\prime \prime}$ satisfies the KMS condition, we have

$$
\begin{aligned}
\varphi_{F}\left(A B\left(f_{0}\right)\right) & =\hat{\varphi}\left(A B\left(f_{0}\right) F\right) \\
& =\hat{\varphi}\left(A F B\left(f_{0}\right)\right)=\hat{\varphi}\left(B\left(f_{\beta}\right) A F\right) \\
& =\varphi_{F}\left(B\left(f_{\beta}\right) A\right) .
\end{aligned}
$$

Hence $\varphi_{F}$ is orthogonal to $A B\left(f_{0}\right)-B\left(f_{\beta}\right) A$ for any $\tilde{f} \in \mathscr{D}$. This implies that the factor components in the central decomposition are $\tau(G)$ invariant and satisfies the KMS condition almost everywhere.

Thus if $\pi_{\varphi}(\mathfrak{A})$ is $\eta$-abelian, the central decomposition of $\varphi$ is (after a possible modification of measure zero components) a unique decomposition into extremal $\tau(G)$ invariant states and the resulting factor states satisfy the KMS boundary condition.

We now come to the converse. If a $\tau(G)$ invariant extremal state $\varphi_{\xi}$ satisfies the KMS boundary condition, then $\pi_{\varphi_{\xi}}(\mathfrak{A})$ is $\eta$-abelian. Let $h_{\infty}$ be an $\mathrm{M}$-filter giving an invariant mean $\eta$. Then we have

$$
\lim _{a} \varphi_{\xi}\left(C_{1}\left[B, A\left(h_{a}\right)\right] C_{2}\right)=0
$$

for each $\xi, C_{1}, C_{2}, B, A \in \mathfrak{A}$, where

$$
A\left(h_{\alpha}\right)=\int \tau(t) A h_{\alpha}(t) d t .
$$

Since

$$
\left|\varphi_{\xi}\left(C_{1}\left[B, A\left(h_{\alpha}\right)\right] C_{2}\right)\right| \leq 2\left\|C _ { 1 } \left|\left\||| C_{2}|\||| B\|||A| \mid\right.\right.\right.
$$

we have by a theorem on bounded convergence

$$
\lim _{\alpha} \varphi\left(C_{1}\left[B, A\left(h_{\alpha}\right)\right] C_{2}\right)=0
$$

for

$$
\varphi=\int \phi_{\xi} d \mu(\xi)
$$

where $\mu$ is a nonnegative measure with the total measure 1 . Hence $\pi_{\varphi}(\mathfrak{X})$ is $\eta$-abelian. Since

$$
\begin{aligned}
& \varphi_{\xi}(A-\tau(t) A)=0 \\
& \varphi_{\xi}\left(A B\left(f_{0}\right)-B\left(f_{\beta}\right) A\right)=0
\end{aligned}
$$

for all $\xi$, we have the same for $\varphi$. Hence $\varphi$ satisfies the KMS boundary condition

Q.E.D. 
Remark. Consider a state $\varphi$ which satisfies the KMS boundary condition and for which $\pi_{\varphi}(\mathfrak{U})$ is $\eta$-abelian.

From the KMS condition, we have

$$
\begin{aligned}
& \mathrm{M} \varphi\left(C_{1}\left[B\left(f_{0}\right), \tau(t) A\right] C_{2}\left(f_{0}^{\prime}\right)\right) \\
= & \mathrm{M} \varphi\left(C_{2}\left(f_{\beta}{ }^{\prime}\right) C_{1} B\left(f_{0}\right) \tau(t) A-B\left(f_{\beta}\right) C_{2}\left(f_{\beta}{ }^{\prime}\right) C_{1} \tau(t) A\right) \\
= & \eta \varphi(\text { the same }) \\
= & \eta \varphi\left(C_{1}\left[B\left(f_{0}\right), \tau(t) A\right] C_{2}\left(f_{0}^{\prime}\right)\right)=0 .
\end{aligned}
$$

Since $B\left(f_{0}\right), B \in \mathfrak{A}, \widetilde{f} \in \mathscr{D}$ is uniformly dense in $\mathfrak{A}$ and the same for $C_{2}\left(f_{0}^{\prime}\right)$, we see that $M\left\{\left[\pi_{\varphi}(A), \pi_{\varphi}(\tau(t) B)\right]\right\}=0$ for any $A, B \in \mathfrak{A}$, in weak sense.

In the course of the above proof, we have obtained

Corollary 3.7. Let $\mathfrak{A}$ be separable and $\varphi$ be a $\tau(G)$ invariant state of $\mathfrak{A}$ satisfying the KMS boundary condition, the central decomposition of $\varphi$ yields factor states which are $\tau(G)$ invariant and satisfies the KMS boundary condition. The same holds for any partial central decomposition (namely the diagonalization of a subalgebra of the center).

Corollary 3. 8. Let $\mathfrak{A}$ be separable and $\phi$ be a $\tau\left(G \times G_{1}\right)$ invariant state of $\mathfrak{A}$ which satisfies the KMS boundary condition with respect to the one parameter group $G$. Further assume that $\pi_{\varphi}(\mathfrak{U})$ is $M$ abelian or $\eta$-abelian for amenable group or weakly asymptotically abelian for a non compact group or large, with respect to $G_{1}$. Then the decomposition of $\varphi$ into extremal $G_{1}$ invariant state yields $\tau(G)$ invariant state satisfying the KMS boundary condition with respect to $G$.

Proof. This follows from the previous Corollary and the known fact that $R_{1}$ is in the center of $\pi_{\varphi}(\mathfrak{U})^{\prime \prime}$ if $\tau_{\varphi}(\mathfrak{U})$ is $M$-abelian or $\eta$-abelian or weakly asymptotically abelian or large.

\section{§4. Non $\boldsymbol{\eta}$-abelian Case}

We now analyze the general structure when $\pi_{\varphi}(\mathfrak{U})^{\prime \prime}$ is not $\eta$-abelian. By Corollary 3. 7, the central decomposition always yields 
a $\tau(G)$ invariant state satisfying the KMS boundary condition, our problem is reduced to a factor state $\phi$ which is $\tau(G)$ invariant and satisfies the KMS boundary condition. For this case we have two steps of possible decomposition towards extremal invariant states. First step is the decomposition according to the central part $R_{1}^{\prime} E_{0} \cap R_{2}^{\prime} E_{0}$. If this part is understood, we may proceed to the case where $R_{1}^{\prime} E_{0}$ is a factor on $E_{0} H_{\varphi}$. These two steps will be discussed here, together with typical examples. The first step necessarily yields a state not satisfying the KMS condition. In the second case, one can find the structure more explicitly. Our example also shows a case where $\pi_{\varphi}(\mathfrak{H})^{\prime \prime}$ is $G$ abelian but not $\eta$-abelian.

Theorem 4.1. Let $R_{a}$ be a sub- $W^{*}$-algebra of $\left(R_{1}^{\prime} E_{0}\right) \cap\left(R_{2}^{\prime} E_{0}\right)$ in $E_{0} H_{\varphi}$. If $R_{a}$ is not contained in $C E_{0}\left(C\right.$ is the center of $\left.\pi_{\varphi}(\mathfrak{H})^{\prime \prime}\right)$ then a decomposition of $\rho$ into $\tau(G)$ invariant states diagonalizing $R_{a}$ necessarily yields some states which do not satisfy the KMS boundary condition.

Proof. Let $\hat{F}$ be a projection in $R_{a}$. Since it is in $R_{2}^{\prime} E_{0}$, there exists a projection $F$ in $R_{2}^{\prime}$ such that $F E_{0}=\hat{F}$. We note that $F$ commutes with $E_{0}$ and $F \in \pi_{\varphi}(\mathfrak{A})^{\prime \prime} \supset R_{2}^{\prime}$. Now we assume that

$$
\varphi_{F}(A)=\left(\Omega_{\varphi}, A F \Omega_{\varphi}\right), \quad A \in \pi_{\varphi}(\mathfrak{H})^{\prime \prime}
$$

satisfies the KMS boundary condition and derive the conclusion that $F$ commutes with any $\pi_{\varphi}(B), B \in \mathfrak{A}$ and hence is in the center of $\pi_{\varphi}(\mathfrak{H})^{\prime \prime}$. (If $\varphi_{F}(A)$ satisfies the KMS condition for $A \in \pi_{\varphi}(\mathfrak{H})$, then it satisfies the same for $A \in \pi_{\varphi}(\mathfrak{X})^{\prime \prime}$ by Corollary 3.3.)

We have

$$
\varphi_{F}\left(A B\left(f_{0}\right)\right)=\varphi_{F}\left(B\left(f_{\beta}\right) A\right) .
$$

We note the previous result (eq. (3.6)) that

$$
\hat{\rho}(A F)=\hat{\rho}(F A), \quad A \in \pi_{\varphi}(\mathfrak{H})^{\prime \prime}
$$

(4.1) implies

$$
\begin{aligned}
\varphi\left(A B\left(f_{0}\right) F\right) & =\varphi\left(B\left(f_{\beta}\right) A F\right) \\
& =\varphi\left(A F B\left(f_{0}\right)\right) .
\end{aligned}
$$


We set $A=A_{1}\left(f_{\beta}{ }^{\prime}\right) A_{2}$. Then we have

$$
\varphi\left(A_{2}\left[B\left(f_{0}\right), F\right] A_{1}\left(f_{0}^{\prime}\right)\right)=0 .
$$

Since $\pi_{\varphi}(\mathfrak{Y})^{\prime \prime} \Omega_{\varphi}$ is dense in $H_{\varphi}$, and $C\left(f_{0}\right)$ is uniformly dense in $\pi(\mathfrak{U})^{\prime \prime}$, we have the desired conclusion:

$$
[B, F]=0, \quad B \in \pi_{\varphi}(\mathfrak{R})^{\prime \prime} .
$$

Remark 4. 2. After the reduction is made by diagonalizing the center of $R_{1}^{\prime} E_{0}$, we obtain $\mathcal{P}$ which is still a trace on $R_{1}^{\prime} E_{0}$ and $R_{2}^{\prime} E_{0}$. Then $R_{1}^{\prime} E_{0}$ and $R_{2}^{\prime} E_{0}$ are now factors of either type $I_{n}$ or type $I I_{1}$. For type $I_{n}$ situation, we have the following theorem. We expect a similar structure for type $I I_{1}$ case.

Theorem 4. 3. If $\Omega_{\varphi}$ is a trace vector on factors of type $I_{n}$, $R_{1}^{\prime} E_{0}$ and $R_{2}^{\prime} E_{0}$, then $\pi_{\varphi}(\mathfrak{A})^{\prime \prime}$ have the following structure:

$$
\begin{aligned}
H_{\varphi} & =H_{1} \otimes H_{2} \\
\Omega_{\varphi} & =\Omega_{1} \otimes \Omega_{2} \\
\pi_{\varphi}(\mathfrak{U})^{\prime \prime} & =Q_{1} \otimes Q_{2} \\
R_{1}^{\prime} & =\mathbb{1} \otimes Q_{2}^{\prime} \\
U_{\varphi}(t) & =U(t) \otimes \mathbb{1}
\end{aligned}
$$

where $U(t)$ is a continuous unitary representation of $G$ on $H_{1}, \Omega_{1}$ is the unique $U(t)$ invariant vector in $H_{1}$ and $\Omega_{2}$ is a trace vector on $Q_{2}$ and $Q_{2}{ }^{\prime}$. If $\varphi$ satisfies the KMS boundary condition, then $R_{2}^{\prime}=1 \otimes Q_{2}$, namely there is no stationary observable in $Q_{1}$.

Proof. We know that $R_{1}^{\prime}$ is isomorphic to $R_{1}^{\prime} E_{0}$. Let $E_{2}$ be the central carrier of $E_{0}$ in $R_{2}$. Then $K=E_{2} R_{2}^{\prime}$ is isomorphic to $R_{2}^{\prime} E_{0}$ and is a weakly closed subalgebra of $R_{2}^{\prime}$. Thus we have two type $I_{n}$ factors $R_{1}^{\prime}$ and $K$ which commutes with each other. Then $\left(R_{1}^{\prime} \cup K\right)^{\prime \prime}$ is a factor of type $I_{n^{2}}$ and $A \rightarrow A E_{0}$ is an isomorphism of $R_{1}^{\prime} \cup K$ onto $\left(R_{1}^{\prime} E_{0} \cup K E_{0}\right)^{\prime \prime}=B\left(E_{0} H_{\varphi}\right)$, where $E_{0}$ is in the commutant of $R_{1}^{\prime} \cup K$. It is easily checked that $\left(R_{1}^{\prime} \cup K\right)^{\prime} \cap \pi_{\varphi}(\mathfrak{H})^{\prime \prime} \equiv \hat{Q}_{1}$ have the property $\pi_{\varphi}(\mathfrak{H})^{\prime \prime}=\hat{Q}_{1} K$. Hence we have the structure of (4.7), (4.9), (4.10), where $1 \otimes Q_{2}=K$ and $Q_{1} \otimes 1=\hat{Q}_{1}$. Since $\varphi$ is irreducible for $\left(Q_{2} \cup Q_{2}{ }^{\prime}\right)^{\prime \prime}$, (4. 8) follows. Since $\phi$ is a trace on $K$ and $R_{1}{ }^{\prime}, \Omega_{2}$ must be a trace 
on $Q_{2}$ and $Q_{2}^{\prime}$. Since $\left(K \cup R_{1}^{\prime}\right)^{\prime \prime} \Omega$ coincides with $E_{0} H_{\varphi}, Q_{2}$ is invariant under $U_{\varphi}(t)$, which implies (4.11), and $\Omega_{1}$ must be a uniqne invariant vector (up to a constant) on $H_{1}$.

We now show that if $\Omega_{1}$ satisfies the KMS boundary condition and some $A$ in $Q_{1}$ commutes with $U(t)$, then $A$ must be a multiple of the identity. First, the uniqueness of invariant vector and $U(t) A \Omega_{\varphi}=\Omega_{\varphi}$ implies $A \Omega_{\varphi}=\lambda \Omega_{\varphi}$ for a scalar $\lambda$. Next we have from the KMS condition

$$
\begin{aligned}
\left(\Omega_{1}, B_{1} A B_{2}\left(f_{0}\right) \Omega_{1}\right) & =\left(\Omega_{1}, B_{2}\left(f_{\beta}\right) B_{1} A \Omega_{1}\right) \\
=\lambda\left(\Omega_{1}, B_{2}\left(f_{\beta}\right) B_{1} \Omega_{1}\right) & =\lambda\left(\Omega_{1}, B_{1} B_{2}\left(f_{0}\right) \Omega_{1}\right) .
\end{aligned}
$$

Therefore, we have $A=\lambda \mathbb{1}$.

Example 4. 4. (4.7) (4.11), where $Q_{2}$ and $Q_{2}^{\prime}$ may be any finite factor, gives an examples, which are not $G$-abelian. In this case any further decomposition with respect to $R_{1}^{\prime}$ which is obviously non unique yields states which differ with respect to stationary observables $Q_{2}$ but which are essentially the same for the time dependent part $Q_{1}$. In general $Q_{1}$ can contain also stationary observables for which $\Omega_{1}$ is necessarily an eigen state.

Example 4. 5. Let $I$ be a finite subset of reals. Let $H_{1}$ and $H_{2}$ be both $L_{2}(I)$, namely the Hilbert space of $\{f(x) ; x \in I\}$ with $(f, g)=$ $\sum f(x)^{*} g(x)$. We define a basis $e_{x}$ by $e_{x}(y)=0$ for $x \neq y$ and $e_{x}(x)=1$. We define $U(t)$ on $H_{\varphi}=H_{1} \otimes H_{2}$ by $U_{t}(t)\left(e_{x} \otimes e_{y}\right)=e^{i t(x-y)}\left(e_{x} \otimes e_{y}\right) . \quad \pi_{\varphi}(\mathfrak{X})$ is defined as $B\left(H_{1}\right) \otimes 1$. We then see that $e_{x} \otimes e_{x}, x \in I$ span $E_{0} H_{\varphi}$. An operator in $B\left(H_{1}\right)$ can be represented by a matrix $\mathrm{A}(x, y)$. Then $R_{1}$ consists of all $A \otimes \mathbf{1}$ for which $\mathrm{A}(x, y)=0$ if $x \neq y$ and the $\mathrm{A}(x, x)$ are arbitrary. $R_{2}$ consists of $1 \otimes A$ with the same $A . \quad R_{1} E_{0}=R_{2} E_{0}$ is abelian and hence we have $G$-abelian property. We set

$$
\begin{aligned}
& \Omega_{\varphi}=\sum e^{-\beta x} e_{x} \otimes e_{x} \\
& \phi(A)=\left(\Omega_{\varphi}, A \Omega_{\varphi}\right)=\sum_{x} \mathrm{~A}(x, x) e^{-\beta x} .
\end{aligned}
$$

Then $\Omega_{\varphi}$ is cyclic for $\pi_{\varphi}(\mathfrak{I})$, invariant under $U(t)$ and $\phi$ satisfies the KMS boundary condition: 


$$
\begin{aligned}
F_{1}(t) & \equiv \varphi(A B(t))=\sum_{x, y} \mathrm{~A}(x, y) \mathrm{B}(y, x) e^{-\beta x} e^{i t(y-x)} \\
F_{2}(t) & \equiv \varphi(B(t) A)=\sum_{x y^{\prime}} \mathrm{B}(x, y) \mathrm{A}(y, x) e^{-\beta x} e^{i t(x-y)} \\
& =\sum_{x^{\prime} y^{\prime}} \mathrm{A}\left(x^{\prime}, y^{\prime}\right) \mathrm{B}\left(y^{\prime}, x^{\prime}\right) e^{-\beta x^{\prime}} e^{i(t+\beta)\left(y^{\prime}-x^{\prime}\right)} \\
F_{2}(t) & =F_{1}(t+i \beta) .
\end{aligned}
$$

However $\pi(\mathfrak{U})$ is a factor and its center is trivial. From our theorem, $\pi(\mathfrak{U})$ cannot be $\eta$-abelian. In fact

$$
\mathrm{M}\{[A, B(t)]\}(x, y)=\mathrm{A}(x, y)(\mathrm{B}(y, y)-\mathrm{B}(x, x))
$$

which is non zero for some $A$ unless $E_{0} B E_{0}$ is a multiple of the identity operator on $E_{0} H$.

\section{References}

[1] Haag, R., N. M. Hugenholtz and M. Winnink, Comm. Math. Phys. 5 (1966), 215236.

[2] Hugenholtz, N. M., Comm. Math. Phys. 6 (1967), 189-193.

[3] Robinson, D., Comm. Math. Phys. 3 (1968), 337-348.

[4] Araki, H., Publ. RIMS Kyoto Univ. Ser. A, 4 (1968), 361-371.

[5] Ruelle, D., Comm. Math. Phys. 3 (1966), 133-150.

[6] Kastler, D. and D. W. Robinson, Comm. Math. Phys. 3 (1966), 151-180.

[7] Doplicher, S., R. V. Kadison, D. Kastler and D. W. Robinson, Comm. Math. Phys. 6 (1967), 101-120.

[8] Doplicher, S. and D. Kastler, Comm. Math. Phys. 7 (1968), 1-20.

[9] St $\phi$ rmer, E., Comm. Math. Phys. 5 (1967), 1-22.

[10] Lanford, E. and D. Ruelle, J. Math. Phys. 8 (1967), 1460-1463.

[11] Araki, H., Progr. Theoret. Phys. 32 (1964), 844-854.

[12] Winnink, M., An application of $C^{*}$ algebras to quantum statistical mechanics of systems in equilibrium, Groningen Thesis, 1968.

Note added in proof :

From (3.7), it follows that $h_{a}$ in the proof of Theorem 3.6. can be taken to be a sequence $h_{n}$ which is $1 / n$ in $[0, n]$ and 0 outside. The authors are indebted to Dr. Winnink for the following comments : Theorem 3.6. follows easily from results in [12]. Theorem 4.1 overlaps with Theorem 3.6. 
